\title{
The Influence of Stanley Mandelstam
}

\author{
Michael B. Green
}

\author{
Department of Applied Mathematics and Theoretical Physics \\ Wilberforce Road, Cambridge CB3 OWA, UK \\ School of Physics and Astronomy \\ Queen Mary University of London, Mile End road, London E1 4NS, UK
}

I hardly knew Stanley Mandelstam - our paths rarely crossed, and when they did our discussions were restricted to technical, rather than personal, issues. It is, however, an honour to be asked to write this short memoir since his work was hugely innovative and he was one of the pioneers who laid the foundations for much of the subject of my research.

My interests as a PhD student in Cambridge in the late 1960's were strongly influenced by the ideas of S-matrix theory that had emerged from Berkeley, largely following the work of Chew, and Mandelstam. Stanley made use of his exceptional understanding of the analytic properties of perturbative quantum field theory to motivate a more rigorous approach to Smatrix theory. This led him to the covariant formulation of scattering amplitudes in terms of "Mandelstam variables" and to the "Mandelstam representation", which provided an elegant framework for discussing dispersion relations. He was one of the early contributors to Regge pole theory and its relation to sums of Feynman diagrams. These were the basic ingredients for much of the S-matrix programme that attempted to explain the strong interactions in the absence of a quantum field theory description.

Stanley was prominent in the series of developments relating to the strong interactions that grew out of the S-matrix programme and were taking place during my period as a graduate student. He realised that the dual relation between resonances and Regge poles necessitates the presence of an infinite number of narrow resonance poles lying on linear Regge trajectories. This was subsequently explicitly realised in Veneziano's 1968 paper that introduced the dual resonance model that was supposed to describe meson scattering and later developed into string theory. Stanley immediately began making seminal contributions to the development of dual models and then to their formulation as string theories. He and his Berkeley group formulated dual model $n$-particle tree amplitudes that included spinning external particles, as well as internal symmetries, which are closely related to the fermionic string theory of Ramond, Neveu and Schwarz.

I visited Berkeley in the Summers of 1971 and 1972 but, to my regret, I did not interact with Stanley. In the Summer of 1973 I participated in a small and very interesting conference on dual models at CERN, in which the highlight was the arrival of preprints of two remarkable papers by Stanley - although he himself did not attend the meeting. These papers demonstrated how to calculate scattering amplitudes in bosonic string theory and in Ramond-Neveu-Schwarz string theory by making use of the light-cone gauge. The lightcone gauge for free string theory had been introduced in a beautiful paper by Goldstone, 
Goddard, Rebbi and Thorn a year earlier and had led to a great simplification in the classification of the spectrum of physical states in dual models. Stanley's two papers provided a meticulous derivation of string scattering amplitudes that involved a detailed discussion of two-dimensional conformal field theory in the presence of suitable world-sheet boundary conditions. The second paper determined the four-fermion amplitude, which had been the subject of much discussion at the conference since the covariant rules for constructing such amplitudes were causing confusion (which was sorted out more than a decade later when the role of world-sheet BRST ghosts was developed by Friedan, Martinec and Shenker). Mandelstam's light-cone formalism bypassed this confusion - in retrospect this is achieved by virtue of the fact that BRST ghosts do not enter in the light-cone gauge treatment.

Stanley's implementation of light-cone gauge methods was hugely influential. They provided a practical method for tackling a variety of otherwise obscure problems. This was particularly relevant to my own research in several contexts. Firstly for studying a possible route to a realistic string theory of hadrons in the late 1970's, and then for understanding the structure of superstring tree and one-loop amplitudes constructed by John Schwarz and me in the early 1980's.

With the advent of QCD and asymptotic freedom in 1973, Stanley turned his attention to properties of Yang-Mills theory and quark confinement, which was a most challenging subject that also had clear connections to a possible string theory description of hadrons. He (as well as 't Hooft) made the crucial observation that the QCD vacuum exhibits a dual Meissner effect, in which colour electric flux is confined into string-like flux tubes by a condensate of magnetic monopoles and vortices.

In 1983 Stanley again made use of the simple properties of light-cone gauge superspace in his proof of the finiteness of $\mathcal{N}=4$ supersymmetric Yang-Mills theory. This (together with a related proof by Lars Brink and collaborators) provided further inspiration for the corresponding superspace formulation of light-cone gauge superstring field theory by Lars Brink, John Schwarz and myself.

Stanley returned to work on string theory in the late 1980's, a period in which I met him more frequently. As ever, he was a mine of information, which was often difficult to understand but always merited careful attention. In 1992 he produced a notable proof of the ultraviolet finiteness of superstring amplitudes to all orders in perturbation theory, which was based on a particularly subtle combination of arguments. I did not feel confident that I understood Stanley's arguments, although I felt confident that Stanley did (as did his ex-students Charles Thorn and Nathan Berkovits with whom I had many interactions). In recent times his proof has been refined and generalised to take into account infrared issues.

I last met Stanley at the conference to celebrate Bruno Zumino's 90th birthday in 2013. It was delightful to find him basically unchanged - quiet as a mouse but as enthusiastic as ever about physics, despite having retired many years ago. He was a remarkably original thinker, who was also exceptionally modest and clearly enjoyed the process of discovery, down to every nitty-gritty detail. 